\title{
A rare chronic myeloid leukemia case with Philadelphia chromosome, BCR-ABL e13a3 transcript and complex translocation involving four different chromosomes
}

\author{
WALID AL ACHKAR ${ }^{1}$, ABDULSAMAD WAFA $^{1}$, BASHAR YOUSEF ALI $^{1}$, \\ MARINA MANVELYAN ${ }^{2}$ and THOMAS LIEHR ${ }^{2}$ \\ ${ }^{1}$ Molecular Biology and Biotechnology Department, Human Genetics Division, Atomic Energy Commission, \\ Damascus, Syria; ${ }^{2}$ Jena University Hospital, Institute of Human Genetics and Anthropology, Jena, Germany
}

Received May 4, 2010; Accepted June 22, 2010

DOI: 10.3892/ol_00000139

\begin{abstract}
The so-called Philadelphia (Ph) chromosome is present in more than $90 \%$ of chronic myeloid leukemia (CML) patients. Around $5-10 \%$ of these patients show complex translocations involving other chromosomes in addition to and/ or besides chromosomes 9 and 22. CML cases with fusion transcripts, such as e13a3, in which ABL exon 3 rather than exon 2 has fused to $\mathrm{BCR}$, are extremely rare. Such reported cases with the e13a3 transcript showed the Ph chromosome on karyotype analysis. This study reported a rare $\mathrm{Ph}$ chromosomepositive CML case with new complex chromosomal aberrations and an e13a 3 BCR-ABL transcript that has yet to be established. A four-chromosome translocation involving chromosomal regions 12p11.2, 19q13.3, 9q34.1 and 22q11.2, besides a trisomy 8 and a derivative chromosome 12, were identified using high resolution multicolor banding. Reverse transcription polymerase chain reaction products showed the presence of BCR-ABL fusion transcript e13a3, and this signifies the major BCR breakpoint. The significance of the observed rearrangements and their possible role in the progression of CML was investigated.
\end{abstract}

\section{Introduction}

Chronic myeloid leukemia (CML) is a myeloproliferative disorder characterized by the expansion of a clone of pluripotent hematopoietic stem cells carrying the chimeric BCR-ABL fusion gene. The Philadelphia $(\mathrm{Ph})$ chromosome created as a result of $\mathrm{t}(9 ; 22)(\mathrm{q} 34 ; \mathrm{q} 11)$ is observed in more than $90 \%$ of

Correspondence to: Dr Walid Al Achkar, Molecular Biology and Biotechnology Department, Human Genetics Division, Atomic Energy Commission of Syria, P.O. Box 6091, Damascus, Syria E-mail: ascientific@aec.org.sy

Key words: chronic myeloid leukemia, variant Philadelphia chromosome, e13a3, fluorescence in situ hybridization, high resolution multicolor banding, reverse transcription polymerase chain reaction
CML patients. The BCR-ABL fusion gene is formed by transposing the 3 ' portion of the ABL oncogene from $9 q 34$ to the 5 ' portion of the BCR gene on chromosome 22 , and this fusion gene encodes a constitutive active tyrosine kinase (1). BCR breakpoints localize on 1 of 3 breakpoint cluster regions (bcr): major bcr spanning BCR exons 12-16, minor bcr spanning BCR alternative exons $2^{\prime}$ and 2 and $\mu$ bcr spanning downstream of exon 19. The majority of the BCR-ABL fusion transcripts are e13a2, e14a2, with ela2 and e19a2 being less common (2). Extremely rare CML cases with fusion transcripts, such as e13a3, in which ABL exon 3 rather than exon 2 is fused to $\mathrm{BCR}$, were previously reported (3-6). Cases reported to have the e13a3 transcript showed the $\mathrm{Ph}$ chromosome on karyotype analysis. A cryptic or variant rearrangement in which the typical $\mathrm{Ph}$ chromosome is not visible at the karyotype level is noted in 5-10\% of CML patients (7). This rearrangement can be found in cases of normal or complex karyotypes. The mechanisms for these rearrangements are difficult to determine. This study reported a rare $\mathrm{Ph}$ chromosome-positive $\mathrm{CML}$ case involving the e13a3 BCR-ABL transcript and new complex aberrations, including four chromosomal breakpoints.

\section{Materials and methods}

Case report. A 25-year-old female was diagnosed as suffering from CML in the chronic phase (CP) after a blood cell count was initiated in November 2009 due to hepatosplenomegaly and loss of weight. The hematologic parameters were: white blood cells (WBC) 122x10\%/1 (96.6\% neutrophils, 1.55\% lymphocytes, $0.170 \%$ monocytes, $1.68 \%$ eosinophiles and $0.04 \%$ basophiles). The platelet count was $156 \times 10^{9} / 1$ and the hemoglobin level was $10.3 \mathrm{~g} / \mathrm{dl}$. No treatment had been administered prior to the test. The patient was referred to our laboratory one month later, after treatment with hydroxyurea $(2,000 \mathrm{mg} /$ day). Thus, the previous relevant symptoms disappeared. The more recent hematological parameters were: WBC 4.5x10 $/ 1$ (42.9\% neutrophils, $44.1 \%$ lymphocytes and $13 \%$ monocytes). The platelet count was $375 \times 10^{9} / 1$ and the hemoglobin level was $9.9 \mathrm{~g} / \mathrm{dl}$.

Banding cytogenetics. Chromosome analysis was performed using the GTG-banding technique according to standard 


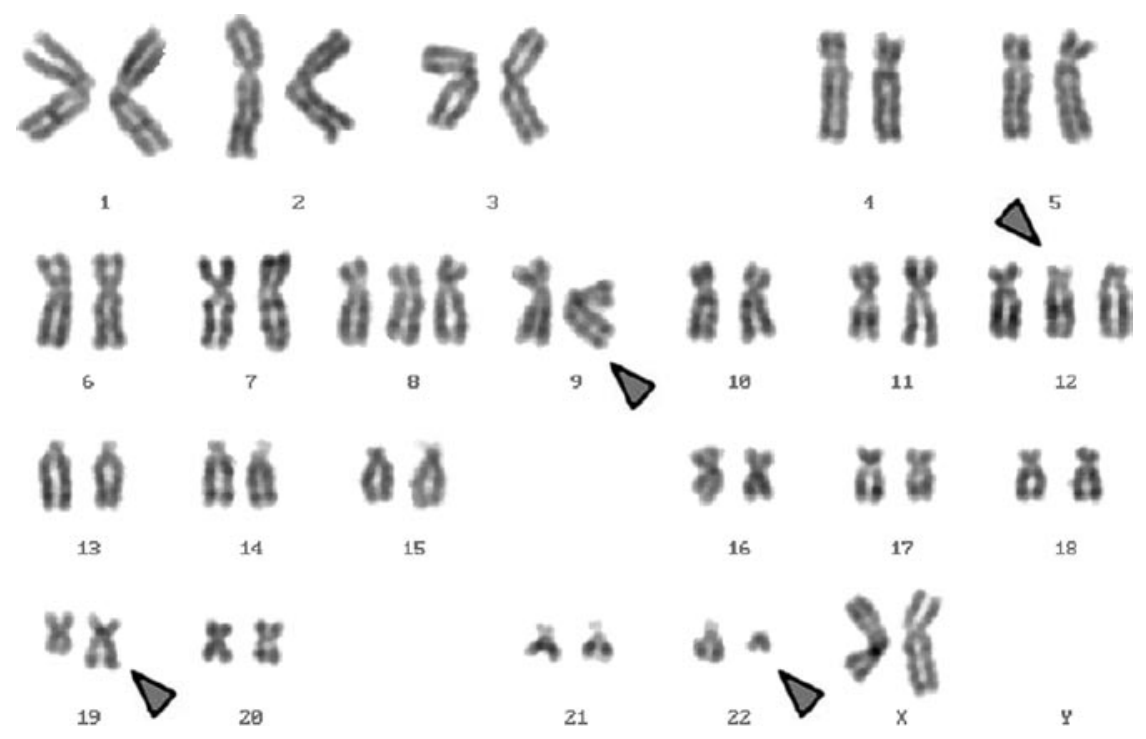

Figure 1. GTG-banding revealed a complex karyotype involving two further chromosomes besides chromosomes 9 and 22. Derivative chromosomes are indicated by the arrowheads.

procedures (8). A total of 20 metaphases, obtained from the unstimulated bone marrow of the patient, were analyzed. Karyotypes were described according to the International System for Human Cytogenetic Nomenclature (9).

Molecular cytogenetics. Fluorescence in situ hybridization (FISH) using a LSI BCR/ABL dual color dual fusion translocation probe (Abbott molecular/Vysis, Des Plaines, IL, USA) was applied according to the manufacturer's instructions. Array-proven multicolor banding (aMCB) probe sets based on microdissection-derived region-specific libraries for chromosomes 9, 12, 19 and 22 were applied as previously described $(10,11)$. A total of 20 metaphase spreads were analyzed, using a fluorescence microscope (AxioImager.Z1 mot, Zeiss) equipped with appropriate filter sets to discriminate between a maximum of five fluorochromes and the counterstain DAPI (4',6-diamino-2-phenylindole). Image capturing and processing were carried out using an Isis imaging system (MetaSystems, Altlussheim, Germany) for the MCB evaluation.

RNA isolation and reverse transcriptase-polymerase chain reaction. Total RNA was extracted from $1.5 \mathrm{ml}$ of fresh peripheral blood immediately after collection using the InviTrap RNA kit (Invitek, Germany). A negative control was used to monitor RNA isolation. RNA concentration was estimated by absorbance at $260 \mathrm{~nm}$ and its purification was determined by a 1.8:2.0 ratio of absorbance values at 260:280 $\mathrm{nm}$. The RNA solutions were stored at $-80^{\circ} \mathrm{C}$. Reverse transcription (RT) for the complementary DNA (cDNA) synthesis, polymerase chain reaction (PCR) and nested PCR amplification of the BCR-ABL gene were performed using a ready-to-use Genequality BCR-ABL kit (AB Analitica, Italy) and a GeneAmp ${ }^{\circledR}$ PCR System 9700 thermocycler. Two positive controls were used to monitor amplification, one with $\beta 2$-microglobuline and the second b3a2 with the patient sample. The negative controls used serial water. PCR products were electrophoresed on an ethidium bromide-stained $2.5 \%$ agarose-TAE-gel and observed under UV light. The type of amplified BCR/ABL
cDNA was established on the basis of the size compared with the molecular markers and the primers used (b3a2, $353 \mathrm{bp}$; b2a2, 278 bp; b3a3, 179 bp and b2a3 (e13a3), 104 bp).

\section{Results}

Karyotyping was performed before and after the initiation of chemotherapy treatment, showing the following karyotypic changes: a complex karyotype 48,XX,+8,+der(12)t(12;19),t(9;12 ;19;22)/47,XX,+8,t(9;12;19;22)/47,XX,+der(12)t(12;19),t(9;12;1 $9 ; 22) / 46, X X, t(9 ; 12 ; 19 ; 22)$ was determined using GTG-banding (Fig. 1) and was further specified by molecular cytogenetic studies (Fig. 2). A dual-color-FISH using a probe specific for $\mathrm{BCR}$ and $\mathrm{ABL}$ showed a typical Ph chromosome with a BCR/ ABL fusion gene. However, sections of chromosome 22 were present on a der(19) (Fig. 2F). A multi-color (M)-FISH, was applied to exclude further cryptic rearrangements (Fig. 2A). Thus, the four chromosomes 9, 12, 19 and 22 were found to be involved. aMCB using probes for the corresponding chromosomes were performed as previously reported (11). A complex translocation among the four chromosomes was detected (Figs. 2B-E), and the following final karyotypes were obtained: 48,XX,+8,+der(12)t(12;19)(p11.2;q13.3),t(9;12;19;22) (q34.1;p11.2;q13.3;q11.2)[8]/47,XX,+8,t(9;12;19;22) (q34.1;p11.2;q13.3;q11.2)[2]/47,XX,+der(12)t(12;19) $(\mathrm{p} 11.2 ; \mathrm{q} 13.3), \mathrm{t}(9 ; 12 ; 19 ; 22)(\mathrm{q} 34.1 ; \mathrm{p} 11.2 ; \mathrm{q} 13.3 ; \mathrm{q} 11.2)$ [2]/46,XX,t(9;12;19;22)(q34.1;p11.2;q13.3;q11.2)[8].

It is likely that due to low chromosomal resolution, the complexity of the karyotype was initially missed. RT-PCR analysis of the fusion transcript showed a band corresponding to the e13a3 (b2-a3) transcript. However, the band was 104 bp (Fig. 3).

\section{Discussion}

We described a rare $\mathrm{Ph}$ chromosome-positive CML case with the e13a3 BCR-ABL transcript and a new complex variant translocation $\mathrm{t}(9 ; 12 ; 19 ; 22)(\mathrm{q} 34.1 ; \mathrm{p} 11.2 ; \mathrm{q} 13.3 ; \mathrm{q} 11.2)$ that was 


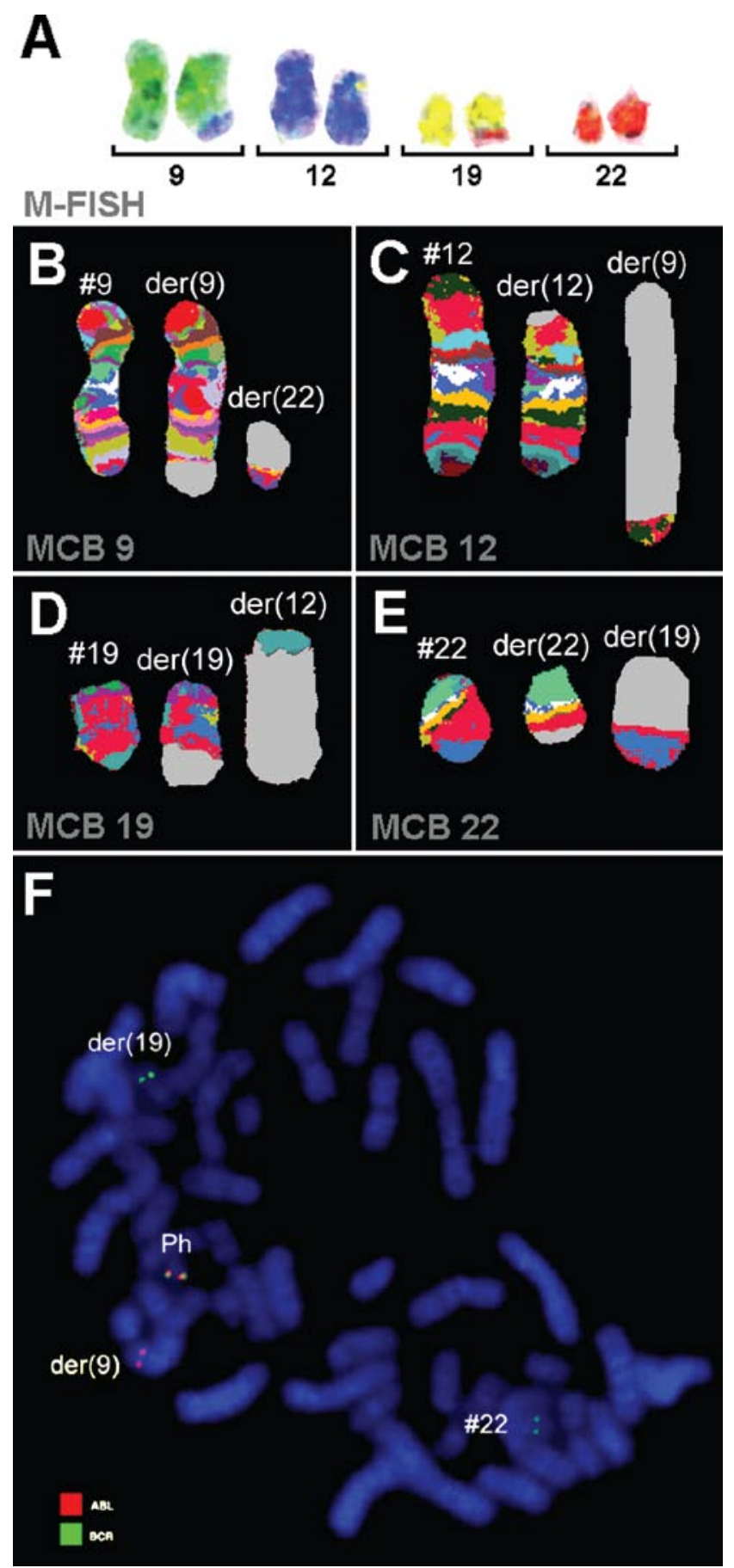

Figure 2. Karyotype and chromosomal aberrations were confirmed using molecular cytogenetic approaches. (A) M-FISH confirmed the complexity of the karyotype: $48, \mathrm{XX},+8,+\operatorname{der}(12), \mathrm{t}(9 ; 12), \mathrm{t}(12 ; 19), \mathrm{t}(19 ; 22)$. (B-E) The application of aMCB analysis using probe sets for chromosomes 9, 12, 19 and 22 is shown. The normal chromosomes are shown to the left of the image, and the derivative of the four chromosomes to the middle and right. Using aMCB probes, the light gray areas show unstained regions on the derivative chromosomes. (F) Fluorescence in situ hybridization (FISH) using probes for BCR (green) and ABL (red) confirmed the involvement of chromosome 19 in the rearrangement present in this case. \#, chromosome; der, derivative chromosome; Ph, Philadelphia chromosome.

detected. Apart from a trisomy 8 and an additional derivative chromosome 12, two additional chromosomal alterations were present. To the best of our knowledge, this translocation has yet to be elucidated in CML (12).

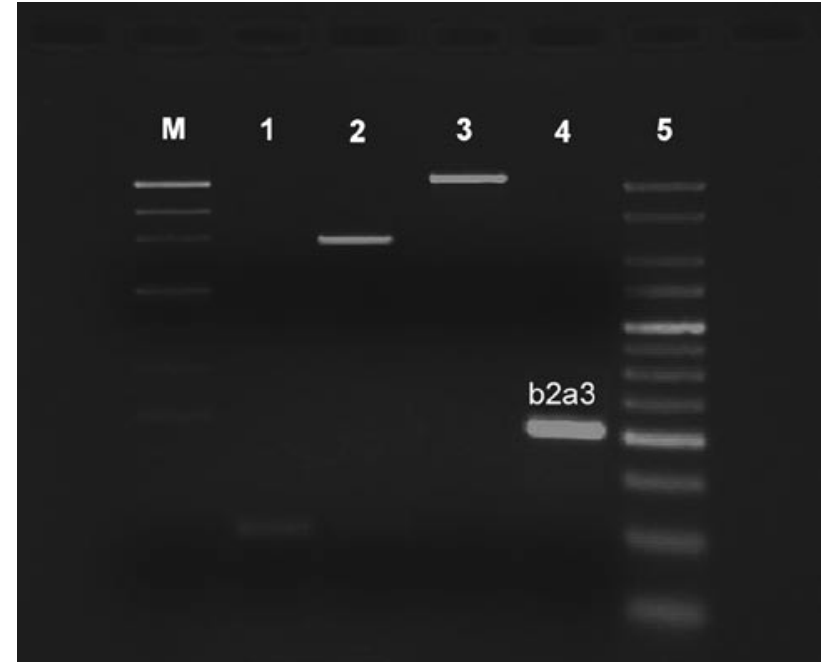

Figure 3. Gel electrophoresis of the nested RT-PCR products. Lane M, 501-110 bp marker; lane 1, negative control; lane 2, positive control (b3a2, $353 \mathrm{bp}$ ); lane 3, $\beta 2$-microglobuline (535 bp); lane 4, BCR-ABL (b2a3, 104 bp) from the patient and lane $5, \mathrm{M}^{1} / 4100 \mathrm{bp}$ molecular weight marker.

In $5-10 \%$ of $\mathrm{Ph}$ chromosome CML cases complex translocations in addition to those and/or besides chromosomes 9 and 22 (13) are noted. At present it appears that in such rearrangements any other chromosome may be involved. However, it has been suggested that the distribution of chromosomes and breakpoints is non-random with the chromosomal bands most susceptible to breakage being $1 \mathrm{p} 36,3 \mathrm{p} 21,5 \mathrm{q} 31,6 \mathrm{p} 21$, 9q22, 10q22, 11q13, 12p13, 17p13, 17q21, 17q25, 19q13, 21q22, $22 q 12$ and $22 q 13$ (7), showing one match with the present case, i.e., $19 \mathrm{q} 13$.

The progression of CML from CP to blast crisis is frequently associated with non-random secondary chromosomal aberrations such as $+8, \mathrm{i}(17 \mathrm{q}),+19$ and an extra $\mathrm{Ph}$ chromosome (14).

Trisomy 12 is the most frequently reported chromosome abnormality in B-cell chronic lymphocytic leukemia (B-CLL). It is found in one third of cytogenetically abnormal CLL by conventional karyotype (15) and in approximately $11-46 \%$ of cases when interphase FISH is used (16).

The majority of CML patients express e13a2 (b2-a2) or e14a2 (b3-a2) of BCR-ABL mRNA encoding for p210 Bcr-Abl tyrosine kinase. These two types are detected by RT-PCR (17-19). CML with the e13a3 transcript is extremely rare, and RT-PCR for e13a 2 and e14a 2 is usually negative because ABL exon 2 is deleted (20). Our case was diagnosed using conventional G-band, FISH, M-FISH, MCB and RT-PCR.

In conclusion, we reported a rare $\mathrm{Ph}$ chromosome-positive CML case in CP with the rare e13a3 BCR-ABL transcript and new complex variant translocation involving the chromosomes $9,12,19$ and 22 .

\section{Acknowledgements}

We thank Professor I. Othman, the Director General of the Atomic Energy Commission of SYRIA (AECS) and Dr N. MirAli, Head of Molecular Biology and Biotechnology Department for their support. This work was supported by the AECS, and in part by the Stefan-Morsch-Stiftung, Monika-Kutzner-Stiftung and the DAAD (D/07/09624). 


\section{References}

1. Shtivelman E, Lifshitz B, Gale RP and Canaani E: Fused transcript of abl and bcr genes in chronic myelogenous leukemia. Nature 315: 550-554, 1985.

2. Deiningger MW, Goldman JM and Melo JV: The molecular biology of chronic myeloid leukemia. Blood 96: 3343-3356, 2000.

3. Al-Ali HK, Leiblein S, Kovacs I, Hennig E, Niederwieser D and Deininger MW: CML with an ela3 BCR-ABL fusion: rare, benign, and a potential diagnostic pitfall. Blood 100: 1092-1093, 2002 .

4. Liu LG, Tanaka H, Ito K, Kyo T, Ito T and Kimura A: Chronic myelogenous leukemia with e13a3 (b2a3) type of BCR-ABL transcript having a DNA breakpoint between ABL exons a2 and a3. Am J Hematol 74: 268-272, 2003.

5. Snyder DS, McMahon R, Cohen SR and Slovak ML: Chronic myeloid leukemia with e13a3 BCR-ABL fusion: benign course responsive to imatinib with an RT-PCR advisory. Am J Hematol 75: 92-95, 2004.

6. Pienkowska-Grela B, Woroniecka R, Solarska I, Kos K, Pastwińska A, Konopka L and Majewski M: Complete cytogenetic and molecular response after imatinib treatment for chronic myeloid leukemia in a patient with atypical karyotype and BCR-ABL b2a3 transcript. Cancer Genet Cytogenet 174: 111-115, 2007.

7. Johansson B, Fioretos $\mathrm{T}$ and Mitelman F: Cytogenetic and molecular genetic evolution of chronic myeloid leukemia. Acta Haematol 107: 76-94, 2002.

8. Al Achkar W, Wafa A and Almedani S: BCR translocation to derivative chromosome 2: A new case of chronic myeloid leukemia with a complex variant translocation and Philadelphia chromosome. Oncol Lett 1: 445-447, 2010.

9. Shaffer L, Slovak M and Cambell L (eds): ISCN: An International System for Human Cytogenetic Nomenclature. S. Karger, Basel, 2009.

10. Weise A, Mrasek K, Fickelscher I, Claussen U, Cheung SW, Cai WW, Liehr T and Kosyakova N: Molecular definition of high-resolution multicolor banding probes: first within the human DNA sequence anchored FISH banding probe set. J Histochem Cytochem 56: 487-493, 2008.
11. Liehr T, Heller A, Starke H, Rubtsov N, Trifonov V, Mrasek K, Weise A, Kuechler A and Claussen U: Microdissection based high resolution multicolor banding for all 24 human chromosomes. Int J Mol Med 9: 335-339, 2002.

12. Mitelman F, Johansson B and Mertens F (eds.): Mitelman Database of Chromosome Aberrations in Cancer. http://cgap.nci. nih.gov/Chromosomes/Mitelman, 2009.

13. La Starza R, Testoni N, Lafage-Pochitaloff M, Ruggeri D, Ottaviani E, Perla G, Martelli MF, Marynen P and Mecucci CL: Complex variant Philadelphia translocations involving the short arm of chromosome 6 in chronic myeloid leukemia. Haematologica 87: 143-147, 2002.

14. Sandberg AA: The Chromosomes In Human Cancer And Leukemia. 2nd edition. Elsevier Science, New York, p151-172, 1990.

15. Juliusson G and Merup M: Cytogenetics in chronic lymphocytic leukemia. Sem Oncol 25: 19-26, 1998.

16. Que TH, Garcia Marco J, Ellis J, Matutes E, Brito Babapulle V, Boyle S and Catovsky D: Trisomy 12 in chronic lymphocytic leukemia detected by fluorescence in situ hybridization: analysis by stage, immunophenotype, and morphology. Blood 82: 571-575, 1993.

17. Rowley JD: A new consistent chromosomal abnormality in chronic myelogenous leukemia identified by quinacrine fluorescence and Giemsa staining. Nature 24: 290-295, 1973.

18. Lifshitz B, Fainstein E, Marcelle C, Shtivelman E, Amson R, Gale RP and Canaani E: Bcr genes and transcripts. Oncogene 2: 113-117, 1988.

19. Hughes TP, Morgan GJ, Martiat P and Goldman JM: Detection of residual leukemia after bone marrow transplant for chronic myeloid leukemia: role of polymerase chain reaction in predicting relapse. Blood 77: 874-878, 1991.

20. Masuko M, Furukawa $T$, Abe $T$, Wada R, Maruyama $S$, Kitajima T, Shibasaki Y, Toba K, Okada M and Aizawa Y: A chronic myeloid leukemia patient with atypical karyotype and BCR-ABL e13a3 transcript caused by complex chromosome rearrangement. Int J Hematol 90: 230-234, 2009. 